\title{
Wolfgang Bernet Eingaben als Ersatz für Rechte gegen die Verwaltung in der DDR
}

In der über 4ojährigen Geschichre des Landes gehören die Eingaben zu den Insrituten, welche dic Staats- und Rechisordnung, dic Rechtspolitik und -dogmatik, davon beeinflußt das Demokracieversrändnis tief prägen. Es ist anzumerken, daß sich in den Eingaben ein Großteil der Kluft manifestiert, die im Rechrsverständnis zwischen DDR und BRD gewachsen ist.

Mit den Eingaben ist der Rechtszweig Verwaltungsrceht besonders verquickt. Seine schicksalhafte Liquidation per ordre durch dic damalige oberste Parteiführung der SED im Jahre $195^{8}$ befindet sich auch mit den Eingaben im engen Konnex. Die Existenz eines funktionierenden Vervaltungsrechts setzt nach tradierten europäischen Maßstäben seit dem vorigen Jahrhundert zumindest die Bindung der Verwaltung an Rechtssätze voraus, die - ob in Normativakten festgelegr oder in Urreilen gesprochen - das Qualifikationsmerkmal „öffenclich \& crfüllen müssen. Den Bürgern ermöglicht das Verwaltungsrecht, ihre subjekriven öffentlichen Rechte durch rechisförmige Verfahren würdevoll, sclbsibewußt, kulcurvoll und demokrarisch durchzusezzen. Ohne an dieser Stelle auf die weitere Ausdifferenzierung der funktionellen Seite des Verwaltungsrechts näher eingehen zu können, soll festgestellt werden, daß die umrissene ursprüngliche Rolle des Verwaltungsrechts mir seincr Liquidation folgerichtig und mit aller Konsequenz beiscitc gefegt wurde. Dicser Befund kann - im leicht abnehmenden Maße - auch nach der allmählichen Reanimation des Verwaltungsrechts und seiner Wissenschaftsdisziplin etwa ab dem Jahre 1972 konstatiert werden. In der theorecischen Arbeit von Verwaltungsrcchtswisscnschaftlem sind die Eingaben in ihrer exorbitant von den politischen Führungsorganen favorisierren Position zugunseen eines rcchesscaallichen Instrumenzariums (Anträge, Rechtsmittcl crc.) zurückgedrängt worden. Vor allem wurde sich bemüht, dic Wirkung der Eingaben weitgehend auf den Arrikel 21 der Verfassung der DDR zurückzuschnciden, in dem sie als ein allgemeines Mirgestaltungsrecht der Bürger verankert wurden. Mchr und mehr haben sich in diesem Verbund Standpunkte in der Litcratur durchgesetzt, die Eingaben nicht als cin nahezu universelles Mittcl zu handhaben, um die Geseczlichkeit zu garantieren. ${ }^{2}$ Damir mußte zumindest das angezieltc Wirkungsspcktrum von Arrikel 103 der Vcriassung der DDR zurückgedrängt werden, der die Eingaben zu hochrangigen Mitteln der Gesctzlichkeitssicherung stilisierte.' Das Gesetz über die Zuständigkeit und das Verfalıren der Gerichte

I Vgl. W. Ulbncht, Die Staatslehre des Marxismus/Lemmismus und ihre Anwenalung in Deuischland (Protokollband der Babelsberger Konferenz), Berlin 1958, 5. 35.

2 Vgl. H. Pohl, G.Schulze, Gerwährletstung der Gesetzlichkeıt bei der Eingabenbearbeitung, in: Neue Justiz 6/1979. S. 247; W. Bernct, A. Schowe, R. Schüler. Für effekervere Verwirklichung des Eingabenreches!; in: Neuc Justrz $9 / 1988, S .282$ ff.

3 Vgl. dic unterschicdlichen Possesonen der Art.21 und 103 der Verfassung der DDR vom 6. April 1968, Geseizblar I, S. 199 i.d.F des Gescizes zur Ergänzung und Änderung der Verfassung der DDK vom 7. Okt. 1974, Grsetzblatt I, \$.432. 
zur Nachprüfung von Verwaitungsentscheidungen same Anpassungsrechisetzung aus dem Jahre 19884 solle auch entscheidend die rocale Wirkungssicht auf die Eingaben als umfassende Mitrel zur Geseczlichkeitssicherung im sensiblen Verwalrungsbercich deutlich einschränken. Der Blick und der Hand́lungsrahmen von Bürgern und Verwaltungen wurde durch diesc Normativakce auf rechtsfähige Kontrolle von Verwalcungshandeln gelenkt. Leider war und ist diesem Gesecz, das in mühevoller - von Vertretern der Rechtswissenschaft geführter - Kärnnerarbeit entstanden isc, kein größerer praktischer Erfolg mehr beschieden, obwohl es entscheidende Anschlüsse für eine reclitsstaatlich arbeitende Vcrwalcung und zur Sicherung der Rechte der Bürger gelegt hat. Allerdings muß deurlich hervorgehoben werden, daß das Bestreben, die Allmachr der Eingaben zurückzudrängen und durch rechtsförmigc Verfahren zu crsetzen, von der Verayaltungspraxis und dem sie entscheidend lenkenden hauptamtlichen Apparat der ehemaligen SED kaum substantiell angenommen worden ist. Vielmehr sollte dic zaghafte Geserzgebung auf dem Gebiet der gerichtlichen Koncrollc von Verwaltungshandeln einige drängende Srimmen aus der Rechtswissenschaft der DDR' limitiert befriedigen sowie - vor allem! - negativer werdende Meinungen auf dem internacionalen Parkett im Rahmen der KSZE - Folgekonferenzen über den mangelnden Rechrsschutz der Bürger in der DDR insbesondere auf dem Gebjet der Verwaltung zum Verstummen bringen. In Wirklichkeit schienen dem Verwaltungsapparat die Eingaben das probate Mittel zu sein, um Bürgerwillen und -wollen in rechesähnliche Bahnen zu gicßen und nach dem paternalistischen Konzept, das Scaat und Verwalrung universell dominierte, von Fall zu Fall zu befriedigen oder nichr.

In der jetzigen Zeit der beginncnden Rechtsangleichung zwischen beiden deutschen Staaten darf allerdings nicht vergessen werden, daß die Eingaben das Mentalverhalten von Bürgern und Verwaltungen der DDR einsclineidend prägen. Durch die Eingaben ist nahezu über vier Jahrzehnte eine (Rechts-)Kultur mitbegründer worden, dic das Abdrifcen vom Rechtsstaatlichkeitsdenken und -handeln mit kennzeichnete. Schließlich muß aus dem Prozeß der Rechesangleichung ein Rechtsropos hervorgebrachr werden, der von der Mehrheit der Menschen in jedem der bisherigen Staatsgebicte angenommen und im Alltagsverhalten interiorisiert wird. Der ticfgreifende politische Umbruch in der DDR scit dem Herbst 1989 hate und konnte solches noch nicht bewältigen. Dic Mülen der Ebenen stehen aber auch auf dicsem Gebiet noch bevor und werden sowohl den in der Verwaltung Tätigen als auch den außerhalb zu ihr stehenden Menschen erfassen. Das Verständnis für Bisheriges, Feinfühligkeit bei Verändcrungen und Augenmaß sind hauptsächliche Handlungsgebore für diejenigen, welche rechrspolitische und -dogmarische Aufgaben zu erledigen haben. Die Skizzierung des status quo auf dem Gebict der Eingaben ist einem Prozeß geschuldet, der im folgenden nachgezeichnet werden soll.

In den Verlassungen der ehemaligen Länder der Sowjecischen Besatzungszone Deutschlands ist das Fingabeninstitut als ein Grundrechr annähernd gleichförmig zu

4 Vgl. Geseız über die Zustandighest und das Verfahren der Genchic zur Nachprufung van Verwaltungsentscheidungen. Gesecz zur Anpassung von Regelungen über Rechesmitel der Burger und xur Festlegung der gerıchelichen Zusrändigkeıt für die Nachprüfung von Vervvaltungsentscheıdungen, Verordnungen zur Anpassung von Regelungen über Rechesmittel der Bürger und zur Festlegung gerschulicher Zuständigke» fur dic Nachpnifung von Verwalungsentschetdungen, santich vom 1d. Dex. Igs\$,

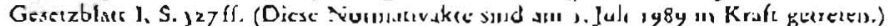

s Vgl.W. Bemes, Gurıcheliche Nachprüfbarkent von Verrvaltungsaleen für die DDR?, in: Wissenschaftli-

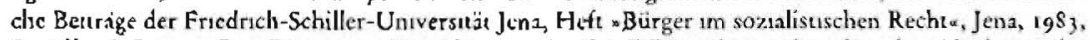
S. 48 ill., E. Poppe, Der Bürger im Verwaltungsrechı der DDR, Sitzungsberichte der Akademie der W/issenschaitien der DDR, 6G/1984; dasselbe U.J. Hcuer, Diskussionsbettrag; K. Wünsche, Zur Einflußnahne der Gerichte auf dic Erhöhung der Rechissicherhere, in: Soxıalisusche Geseczlichkeut. Siclierheıt und Ordnung bei der Entfalung der gescllschafilichen Truebkrälte. Berlin 1985, S. 39 ff. 
vormaligen Eingaben-(Petizions-)Regelungen in deutschen Ländern der Weimarer Republik als auch der Weimarer Reichsverfassung selbst verankert worden. Exemplarisch hieß es in der Verfassung des Landes Thüringen vom 20. Dezember 1946: - Jeder Bürger hat das Recht, Eingaben an den Landrag und an alle Volksvertrecungen zu richrena (Art. 3 Abs. 4). ${ }^{6}$ Damit folgten die Landesverfassungen den deutschen verfassungsrechelichen Traditionen bis in das 19. Jahrhunder hinein. Allerdings muß angemerkt werden, daß das Petitionsrecht des 19. Jahrhunderrs ein vom Bürgertum häufig erstritcenes und vom aufgeklärten Feudalismus schließlich zugestandenes Grundrecht gewesen war.

Die Verfassung vom 7. Oktober $1949^{7}$ setzte in Art. 3 mit der Gescaltung der Eingaben den Duktus der Regelungen der Länderverfassungen fort. Rechtspolitisch von erheblicher Bedeutung ist jedoch, $\mathrm{d}_{a} \beta$ in der chemaligen sowjetischen Besatzungszone Deutschlands und späteren DDR das Institut der (förmlichen) Rechtsmitrel bei Verwaltungshandeln sorvohl gesetzgeberisch als auch in der Verwaltungspraxis weiter cxisticrte. ${ }^{\circledR}$ Damit linder sich auch auf diesem Gebiet ein hohes Maß an Kontinuität zu früheren deutschen Verwaltungsrechtstheorien und -praktiken. Ein Ausdruck dieses Befundes ist die Fortgeltung in novellierter Form der Landesverwaltungsord́nung für Thüringen, in der in vorbildlicher Symbiose Verwaltungsverfahren- und Verwaltungsprozeßrecht vereinigt waren. ${ }^{9}$ Allerdings wurde die in der Landesverwaltungsordnung für Thüringen geregelte und sehr wirksam gewordene Verwaltungsgerichtsbarkeit im Jahre 1948 faktisch stillgeicgt. Dieser vielsagende Vorgang kann hier nicht näher besprochen werden. Auf alle Fälle war damit ein beträchrlicher Tei\} des Rechrsschurzes der Bürger in Verwaltungsangelegenheiren abgebrochen worden. Verblieben waren allerdings - in allen Ländern der Sowjetischen Bcsatzungszone Deurschlands und spätcren DDR - die Rechrsmitrel mit ihren Verfahrensvorschrifeen gegen Verwaltungshandeln. Dic Eingaben erfüllten ihre Wirkungspotenz als ein allgemeines Beschwerdeinstitut gegen Zustände oder Handlungen, die staatliche Organe zu vertreten haben, soweit kein Verwaltungshandeln mit (förmlichen) Rechtsmitteln vorgeschrieben ist.

Einen Einschnit dieser Konzeprion, vor allem in der praktischen Handhabung der Verwaltung selbst, brache die Vorschlags- und Beschwerdeverordnung vom 6. Februar 1953..$^{10} \mathrm{Mit}$ ihr wurde im Grunde versucht, ein universelles Beschwerderecht für die Bürger einzuführen, unablängig davon, ob konkretes Verwaliungshandeln (Verwaltungsakt etc.) vorlag oder nicht. Die Parallele hierzu bicret bis in die heurige Zeit der sowjetische Rechtszustand auf diesem Gebiet. Das sowjetische Recht kennt iraditionellerweise kaum eine Differenzicrung von (förmlichen) Rechrsmitteln der Bürger und Eingaben. Das ist aus der Rechtsgeschichte des zaristischen Rußlands maßgeblich zu erklären, das dic Idee der Rechtsstaatlichkeit im Vertvaltungshandeln nach west- und mitreleuropäischem Gedankengut kaum rezipierte. Das zaristische

6 Vgl. Regierungsblan fúr "Thürngen Teil I 1947, Nr. t vom 23. Jan. 1947, S. I-1; lm Kontext vgl. Veriassung der Provinz Sachsen/Anhalt vom 10. Jan. 1947, Gesetzblatı der Provinz Sachsen/Anhalt Teil L, 1947 Nr.2/3 vom 18. Jan. 1947, S.9-16 (Art. 11): Verfassung des Landes Mecklenburg vom i6. Jan. 1947, Reguerungsblatu fur Mccklenburg 1947 Nr. 1 vom 12. Mirz. 1947, S. 1-8 (An. 10); Verfassung für die Mark Brandenburg vom 6. Feb. 1947. Gesclx- und Verordnungsblat der Provinzialregierung Mark Brandenburg, 3. Jalirgang, Hefe 3. vom 6. Feb, 1947, S. $45-49$ (Art. 2 Abs. 2); Verfassung des Landes Sachsen vom 28. Feb. 1947, Gescrz, Belehle. Verordnungen, Bekanntmachungen, veröffentliche durch die Landesregicrung Sachsen. 3. Ig. Nr. g vom 15. Märx 1947, S. 103-108 (Art. 11 Abs. 2).

7 Vgl. GBl. 19+9, S. 5.

8 W. Menzel, Das Vorschlags- und Beschwerderecht der Werktaingen in der Deutsche'n Demokmuschen Republik, Berlin 1956.

9 Landesverwaliungsordnung vom 10. Jum 1926, Gesetzessammlung, S. 177ff. in der Fassung vom 26. Nov. 1945, Gesetzessammlung 1946, S. 33 ff.

10 Verominung über die Prüfung von Vorschlägen und Beschwerden der Werktätıen vom 6. Feb. 1953, GBI. S. 26jil. 
Reche kannte weder die ausgeprägte Möglichkeir, daß sich die Bürger mis Rechtsmitzeln gegen Entscheidungen der Behörden wenden konnten, noch eine Verwaltungsgerichtsbarkeit nach mittel- oder westeuropäischem Vorbild. Die Errichtung der Sowjetmacht half diesem Zustand wenigstens insoweit ab, als ein allgemeincs Beschwerderecht gegen jegliches Verwaltungshandein konstituiert wurde." Die Vorschlags- und Beschwerdeverordnung von 1953 orientierce sich cindeutig auf allgerneine Vorschläge und Beschwerden der Bürger. Sie hat dic auf Rechrsstaatlichkeit orientierten Rechtsmitcel aber nicht ausdrücklich abgeschaffr. Die Rechtswissenschaft der DDR dicser Jahre hat diesen entscheidenden Punkt erkannt und versuchte zu vermitteln. K. Hönl folge der tradiercen deurschen Aufgliederung des Beschwerderechrs in Reclissmittelbesciswerden und Eingabenbeschwerden und folgcre, daß die (förmlichen) Rechtsmittel nichr Gegenstand der Überlegungen zur Vorschlags- und Beschwerdeverordnung von 1953 seien können. ${ }^{22}$ Dagegen führt W. Menzel folgenden Standpunkt an: "Grundsätzlich getten die Vorschrifren der Vorschlags- und Beschwcrdeverordnung auch für förmliche Rechtsmittel. Enthalten Gesetze oder normakive Verwaltungsakte für die Behandlung von Reclussmitteln Vorschriften, die einzelnen Vorschriften der Vorschlags- und Beschwerdeordnung cntgegen stehen, so gehen jene insoweth, aber auch nur insoweit, diesen vor. Den Normen gegenüber, die das Verfahren für die Behandlung förmlicher Rechrsmitcel regeln, hat die Vorschlags- und Beschwerdeverordnung die Stellung einer lex generalis. $x^{\prime 3}$ Immerlin blcibt auch bei Menzel's Vorstellung die Idee der Eigenständigkeit der Rechesmittel noch erhalten. Das wurde auch dadurch gestützt, daß rechtsnormsetzende Staatsorgane, wie Volkskammer, Ministerrat, Minister u.a., zwar teilweise mir ungerechrfertigten differenzierten Verfahrensvorschrifren, aber immerhin nach tradierten Vorstellungen, Rechtsmittelregelungen erließen.

In der Praxis erfolgre jedoch eine weitgehende Demonrage der Rechismittcl. Nacl und nach wurden sie zu allgemeinen Beschwerden in Sinne der Beschwerdeverordnung von 1953 umfunktionicrt und entsprechend behandelt. Das hat insofern seine tiefsitzenden Spuren hinterlassen, weil das Rechusbewußtsein bci Verwaltungsorganen und Bürgern für stringente Fristen, Instanıenzüge, Formen, Begründungen etc. mehr und mehr verloren ging. In den fünfziger Jahren hat sich ein allgemeines, rechesstaatlich nicht geprägtes Eingabenberwußtsein in der DDR herausgebildet. Das kam den Verwaltungsfunktionären insofern entgegen, weil sie ohnchin über cine stark abnehmende juristische Spezialbildung verfügten und für die oben angegebenen Verf́ahrenselemente immer weniger Verständnis aufbrachsen. Im Grunde wurden die Verfahrensclemente im Verwaltungsrechr besonders negativ stigmatisiert und mir dem Erikerr des Bürokrarismus versehen. Das vermeintlich Neue, Einfachere der Beschwerdeverordnung wurde benuzz, um eine weitergehende Rechtsentleerung der Verwaltung zu befördern. Die relative Ungebundenheir des Beschwerderechis an Verfahrensvorscluriften gaukelte den Bürgern freundliches Verhalten der Verwalcung vor, das sich als weit verfehic crwies. Übrigens trafen dic negativen Aussirkungen der Beschwerdeverordnung von 1953 die Verwaltungsorgane selbst. Mit ihr war nämlich de facto der rechtsstaarlichc Grundsatz verworfen worden, daß cinc endgültig entschiedene Sache im Regelfall nicht wieder angefochten werden darf. Das wurde immer weniger akzepriert, indem millionenfacla gegen (scheinbar)

I1 Aligensein gilt als Beginn der geseizlichen Regelungen des allgemenen Beschwerdercehts die Praklamaton des VI. Sowjetkongresses von 1918 mit dern Aufruf "Uber die strikte Benchtung der Gesetzer, vgl. V. V. Malkow Sowjetskojc zakonodabielsbwo o schalobach I zajav\}enijach. Moskwa 1067. S. 17.

12 K. Hönl, Die Eingaben der Werktätngen als Mittel der Demokratisserung der Arbestsweise des Sinatsapparates, in: Staat und Recht 6/1953, S. 708 I.

1) W. Menzel. (Fn. 8). 
endgültige Verwaltungsentscheidungen immer wieder mit Eingabenbeschwerden vorgegangen wurde.

Der im Jahre 1960 neu gebildetc Staatsrar der DDR secztc die in den fünfziger Jahren begonnene Politik bezüglich der Rechisenteerung der Verwaltung fort und kultivierte das Eingabenrechr. Der Eingabenerlaß vom 27. Febr. 1961's ist bereits von der Normierung her, aber auch in der praktischen Handhabung ein beredter Ausweis für diese Feststellung. In der rechtswissenschaftlichen Literatur der Zeit wird diese Feststellung abgestürzt. So wird direks zum Ausdruck gebracht, daß die individuelle Beschwerde der Bürger (also das förmliche Rechtsmittel) den Charakter von gesellschafelichen Anliegen annehmen müsse (also zur Eingabe werde)." Diese Gegensetzung hat a priori den Verfasser einer Beschwerde mit der Überschrift Eingabe zum Mitgestalrer befördert und den individuellen Beschwerdeführer zum egoistischen Individualisen degradiert. Daraus ist leiche ausrechenbar, was die Bürger der DDR über die Beschwerde an Staatsorgane zumeist als Deklaration anbrachten.

Die Eingaben haben aber durchaus noch in weitere Bereiche hinein gewirkt, die selbst nicht zur Verwaltung gehörten. W. Hafemann har die Eingaben insbesondere für die Durchserzung des sogenannten Produkrionsprinzips hervorgehoben. Das Produktionsprinzip war ein Vorgänger für das Experiment des neuen ökonomischen Systems der Planung und Leitung der Volkswirtschaft, das nach 1968 abgebrochen wurde. Das Produktionsprinzip und das neue ökonomische System der Planung und Leitung der Volkswirtschaft sollten die Arbeitsproduktivitär der DDR mit ökonomischen Stimuli befördern und administrative Einflüsse zurückdrängen. Hafemann berichtet, daß aus dem Bereich VVB Baumwolle bekannt wird, daß sich zahlreiche Eingaben mit betrieblichen Problcmen befassen. Das betrifft folgende Sachfragen: Arbeitsorganisation, sozialistischer Wectbewerb, neue Technik, Erfindungs- und Vorschlagsivesen, Lohn-Prämiensystem, Macerialversorgung, qualitätsgerechte Produktion, Qualifizierung usw. ${ }^{16}$. Hier findet sich die grenzenlose Ausuferung des Sinns der Eingaben. Sie wurden zu universellen Mitteln umfunktioniert, um in allen Bereichen des Lebens einzugreifen. Den Bürgern, den in Verwaltungen, Betrieben und anderen Bereichen Tätigen wurde suggerier, daß die Eingaben wirksame Mirtel sind, um Mißstände zu lösen. Sicher ist durch diese Orientierung manches Problem auf den Tisch gekommen, aber in Grundpositionen ist niemals wirklich cenvas gelöst worden. Hafemann berichter diese Auswucherung auf die Produktionssphäre selbst: "Sie (eine Arbeiterin - W. B.) legte dar, daß in ihrem Betrieb grundsätzliche Mängel bei der Einführung einer neuen Zwirntechnologie bestanden. Das Werk wurde mit Maschinen ausgerüster, dic nicht produktionsreif waren, die geplanten Leistungskennziffern nicht erreichten und die Garnqualizät beeinträchtigten. Trotzdem sollen weitere Werke mit diesen Maschinen ausgerüster werden. " ${ }^{17}$ Eingaben wurden so zum Allheilmitcel gegen nahezu alle möglichen Unzulänglichkeiten erhoben. Selbst Beschwerden über ungeserzliche Enclohnung oder Prämierung der Arbciter in Betricben wurden zu Eingaben crklärt, is auch wenn schon damals eindeutige Rechtsvorschriften über die Schlich-

I4 Vgl. Erbaß des Staatsrates über die Eingaben der Burger und ihre Bearbeıtung durch die Staatsorgane vom 27. Feb. 1961, GB1. I 196x, S. 7.

1) Vgl. z.B. W. Menzel, Der Erlaß des Seaatsrates ülxer die Eingaben der Bürger - en Minel zur Verwirklichung der Programmatuschen Erklarung des Vorsizzenden des Sunusrates der Deutschen Demokratuschen Republik, in: Sta2t und Reche 10/1961، S.18. 57 fl.

$16 \mathrm{Vgl}$. W. Hafemann, Dic Eingabenbearbeitung - Bestandicil der Lcitung der Wirtschait nach dem Produktionspnnzip, un: Sizat und Recht $5 / 1964, S .793$.

17 Vgl. W. Hafemann (Fn. 16), S. 736

18 W. Halemann (Fn. 16). S.801 und 80 ). 
tung von Arbeitsrechtsstreitigkeiten bestanden und gerichrliche Kompetenz luer\}ür existierte.

In der Tat hatte diese von Theoretikern abgestützte (Rechts-)Politik ein Anscliwellen der Eingaben zur Folge, das als ein posiciver Gradmesser für die Qualitär der sozialistischen Demokratie betrachter wurdc. In der Verwaltung der DDR hat bis in das Jahr 1989 zu einem beträchtlichen Teil noch die Devise bestanden: Je mehr Eingaben der Vervaltung zugehen, desto höher ist der Vertrauensgrad der Bürger. Lange Zeit hat sogar ein Wetrbewerb unter Verwaltungsorganen um die höchste Eingabenquote stattgefunden. Die allerorten mit großem Aufwand angeferigten Eingabenanalysen der Verwalcungsorgane belegen, daß tassächlich millionenfach Eingaben eingereiche wurden. Die Erfolgsquote sank jedoch von Jahr zu Jahr beträchtlich, was wicderum eine Vielzahl von Eingaben auslöste. Am Jahresende 1988 waren allein beim Staatsrat der DDR über 100000 Eingaben registriert. Eine eigene Untersucluung hat ergeben, daß mindestens jeder 10. Bürger der DDR pro Jahr eine Eingabe verlaßte. Die Unsinnigkeit hinsichrlich der Hascherei nach hohen Eingabequoten soll folgendes Beispiel belegen, das Untersuchungen von Praktikanten des Verfassers dieses Beitrages von eincm Rat der Stade (Stadt-Kreis) einbrachten:

Das Standesamt des Rates der Stadt hat unter anderem die Befugnis, EheschlieBungsanträge anzunehmen und Ehen zu schließen. Das Amt hat dies offensichtlich langjährig ohnc Beanstandung im Verbund mit seinen anderen Aufgaben wahrgenommen. Bei turnusmäßigen Auswertungen der Eingabenstatistik gab es folghich stecs hinsichtlich der Eingaben durch das Standesamt Eehlanzeigen. Das hatte einige Male zu harscher Kritik an der Lciccrin des Sandesamtes geführt, mic der Begründung, daß fehlende Eingaben ein mangelndes Vertrauensverhältnis der Bürger zum Verwaltungsorgan signalisierten. Um wciteren Kritiken zu entgehen, wurden infolge sämtliche Eheschließungsanträge als Eingaben gemeldet. Damit war das Amt aus der kritischen Zone der Betrachtung heraus, ohne daß ein crkennbarer Nutzen der Handlung zu verzeichnen gewesen wäre.

In einem anderen, auch von Prakrikanten ermitcelten Fall zeugt bereits die Höhe der Eingaben von der Sinnentlecrung dieser Institute.

In einem Stadtkreis von ca. 100000 Einwohnern ballten sich die Eingaben aul dem Gebiet des Wohnungswesens. In dem berreffenden Fachorgan (Dezernat) des Rates der Stadt waren in einem Jahr 21000 Eingaben registriert. Jede Nachfrage in einer Wohnungsangelegenheic, jeder belangarme Anruf in einer Wohnungssache wurde als Eingabe registriert.

Dic für eine fachgerechte Verwaltung notwendige Differenzierung in Anträgc, Rechtsmittel ctc. isc nahezu völlig verloren gegangen. Erst in den letzten Jahren sind einige dieser Dinge im Zusammenlang mic der Resticuierung des Verwaltungsrechts und seiner Wisscnschafesdisziplin wieder geordnee worden. Das fand jedoch keinen durchgehenden Effolg in den Verwaluungsorganen selbst, die nach alchergebrachten Schablonen arbeiteten und nahezu sämtliche Beschwerden, Anträge, Rechtsmittel etc. als Eingabe registrierten. Die wirkliche Bearbeitung von Eingaben und Rechtsmiteln erfolgte immer weniger fachgerecht.

Im Jahre 1969 faßce der Statsrat der DDR den Eingabenerlaß neu. " Offenbar war hinsichtlich der Höhe der Eingabenquote cines bestimme Schmerzgrenze erreicht. Deshalb war im $\$ 2$ Abs. 3 des Erlasses seit vielen Jalıren das crste Mal wieder darauf verwiesen worden, daß die in Rechtsvorschriften vorgesehenen Rechtsmittel keine Eingaben sind. Glciches wurde Ncuerervorschlägen und Anträgen zugescanden,

$19 \mathrm{Vgl}$. Erla des Stazesrates der DDR über die Bearbeıtung der Eingaben der Bürger vam 20. Nov. 1969, GBI. IS. 239 ff. 
deren Bearbeitung durch besondere Rechtsvorschriften geregelt ist. M. Gerlach, damals im Staatsrat für dic Eingabenproblematik zuständig, brachte zum Ausdruck, $\mathrm{daß}$ die Rechtsmittel uneinheitlich geregelt sind und dies für die staatliche Arbeit hemmend wirkt. ${ }^{\text {xo }}$ Infolge dieser neuen Sicht wurden darauthin die in Rechtsvorschriften der DDR enthaltenen Rechtsmittelregelungen hinsichelich der Verfahrenszüge vereinheitlicht." Gelungen ist bis heute keine allgemeine Verwalungsverfahrensordnung für die DDR, in der die Rechrsmittelverfahren aufgehoben sind. Diese Vorgänge zählen zu den Halbherzigkeiten, die die politische Führung des Landes fabrizierte, um einerseits gewissen internationalen Gepflogenheiten im eigenen Land zu entsprcchen, andererseits sich aber in Wahrheit nicht wirklich rechtsstaatlichen Gebocen zu unterzichen. In praxi ist jedenfalls das Gemenge von Eingaben und Rechesmitreln nicht ausgestanden worden. Im Gegenteil! Mit dem Fortgang der Generationen, der Auszehrung der Verwaltung von nahezu jedem juriscisch gebundenen Handeln, des Aufgebens sachlicher Handlungskompctenz der Verwaltung, der Dominanz des Politischen vor dem Juristischen und anderen Faktoren ist dieser Gegensatz noch schärfer hervorgerreten.

Der Eingabenerlaß von 1969 haute - wie so oft in der Geserzgebung der DDR ohne vorherige theoretische Vorbereicung in Form der Beschwerdeausschüsse neue Instanzen geschaffen, um bestimmte Streitigkeiten zwischen Vervaltung und Bürgern zu schlichten. Die Beschwerdeausschüsse waren in den $\$ 20-26$ des Erlasses von 1969 geregelt. Sic erfüllten cin Verfassungsgebor aus Art. 105 der Verfassung von 1968. Bereits die Verfassungsdiktion war nicht eindeutig, ob die Beschwerdcausschüsse als zusätzliche Instanzen zur Schlichrung von Eingabenbeschwerden oder auch zur weiteren Behandlung in Rechrsmitclbeschwerden herangezogen werden konnten. Der Artikel 10s Abs. I der Verlassung ging von einem einheiclichen Oberbcgriff der Beschwerde aus und lauter: "Für Beschwerden gegen Entscheidungen örtlicher Staatsorgane ist der Leiter des Organs zuständig, welches die angefochtene Entscheidung getroffen hat. Ändert der Leiter die Entscheidung nicht, isc der Bcschwerdeführer berechrigi, sich an den Beschwerdeausschuß der zuscändigen Volksvertretung zu wenden.*

Beschwerdeausschüsse wurden gemäß Eingabenerlaß von 1969 bei folgenden örclichen Volksvertretungen gebildet: Bezirkstage, Kreistage, Stadrverordnetenversammlungen und Stadtbezirksversammlungen der Stadtkreise. Zur Bildung der Beschwerdeausschüsse waren ausdrücklich die Volksvertretungen der jeweiligen Ebenen befugl. Der Verfasser dieses Aufsatzes hat damals in Vercin mit anderen Fachkollegen für die juriscische Qualifikarion dieser Ausschüsse gewirkt und um ihre Arbeitstähigkci gefochten. Aus dieser Tätigkeit lassen sich für das ratsächliche Wirken Schlußfolgerungen zichen, dic im Maßstab der DDR durchaus als repräsentativ gelten können, zumal in der rechtswissenschaftlichen Lireratur des Landes diese Ausschüsse kaum abgehandelt wurden.

Zunächst waren die Beschwerdeausschüsse der einzelnen Scufen zuständig für Beschwerden gegen Maßnahmen oder Entscheidungen von Verwaltungsorganen, die den jeweiligen Volksvertrecungen bzw. dom jeweiligen Rat (der Volksvertre-

20 M. Gerlach, Eingaben der Bürger - Eine Form der Beteiligung des Volkes an der Machrausübung, in: Schnitreihe des Staztsrates der Deutschen Demokratischen Republik. Heft 10, J. Wablpenode 1969. S. igl.

21 Vgl. Gesciz uber die Neufassung von Regelungen iber Rechesmittel gegen Entscherdungen stzaclicher Organe vom 24. Junı t971 GBI. 1, S. 49.

Verordnung über die Neufassung von Regelungen über Rechismutel gegen Entrcheidungen seanelicher Ongane vom 24. Jun 1961, GBI. II, S. 463 .

Gleiches ist in Ordnungen geschehicn, in denen Ministur Rechesmited in Rechesvorschrilten neu laBen. dic ste fur ihren Bereich hervorgebrache haten (Kultur. Gesundheieswesen etc.) 
tung) unterseellt waren. Im Normalfall mußte also vor Einschaltung des Beschwcrdeausschusses ein Eingabenverfahren im Verwalrungsapparat negativ für den Bürger abgelaufen sein. In praxi haben Beschwerdeausschüsse diesen Weg allerdings zu Gunsten des Bürgers umgangen. Reichre zum Beispiel ein Bürger seine Eingabe an den Ausschuß, so war es üblich, daß sich dieser der Sache annahm, sic behandelte und eine Entscheidung fällte. Folgte der Beschwerdeausschuß dem Anliegen des Einreichers der Beschwerde, so konnte er folgende wesentliche Entscheidungen treffen:

a) eine Entscheidung über die Sache durch den zuständigen Rar beantragen und dazu entsprechende Enpfehlungen geben;

b) die angefochtene Maßnahme oder Entscheidung über einen offensichtlichen Verstoß gegen die Gesetzlichkeit aussetzen und vom Vorsizzenden des zuständigen Rates ihre unverzügliche Auflebung verlangen.

Die Ausschüsse hacten keine Möglichkeit, in der Sache selbst positiv zu entscheiden. Das war allein wiederum dem Verwaltungsapparat zugestanden. Auch gab es innerhailb des Systems der Beschwerdeausschüsse keinen Instanzenweg.

Die Beschwerdeausschüsse waren aber auch in das Rechtsmittelverfahren eingeschalter. Das läßr sich nicht aus der Verfassung, sondern lediglich indirekt aus $₫ 22$ Abs. 2 Eingabenerlaß crkennen, in dem es heißr, daß die geserzlich vorgesehenen Rechtsmittel vor $\delta \mathrm{cm}$ Tärigwerden des Beschwerdeausschusses in Anspruch genommen werden solJen. Herausgehoben werden muß, daß die Beschwerdeausschüsse ausschließlich Beschwerden (Eingaben und Rechtsmittel) des exekutiven Verwaltungsapparares der Volksvertrecungen beliandeln durften. Beschwerden gegen die Deursche Volkspolizei waren darin selbstverständlich nicht enthalcen. Als völlig unzulänglich erwies sich auch der Umstand, daß Beschwerdeausschüsse erst ab der Ebene der Kreise gebildet wurden, also nicht bei Volksvertretungen der (kreisangchörigen) Städre und Gemeinden. Eigene Untersuchungen haben ergeben, daß die Beschwerdeausschüsse in den durchgängigen Jahren ihres Bescehens sehr wenig von den Bürgern angerufen wurden. Der Untersuchungszeitraum beträgr die Jahre 1970/ 197 X/1972/1973. Untersucht wurden Beschwerdeausschüsse von Stadevcrordnctenversammlungen der Stadt-Kreise und Kreistage der Landkreise. ${ }^{22}$

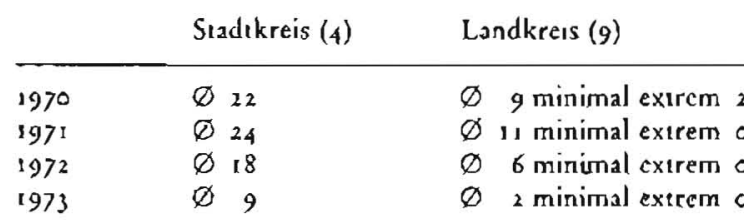

Das Gcsamtergebnis der Frequentierung der Ausschüsse durch die Bürger war zunehmend negariv. Ursachen waren u.a. mangelnde Kompetenz zur Streitentscheidung in Verwaltungsangelegenheiten, weitgehende Ressentiments des exekutiven Apparats gegen diese Organe, abnehmende Popularität bei den Bürgern.

Die Beschwerdcausschüsse spielen nochmals eine Rolle bei der Schaffung des Geserzes über dic örtlichen Volksvertretungen in den Jahren 1972/73. Im Geserzentwurf $(\$ 16)$ waren diese Gremien noch enthalten. ${ }^{23}$ Insbesondere massive Hinweise aus dem Verwaltungsapparac führten dazu, daß sie im verabschiedeten Geserz vom 12. Junj $1973^{\text {24 }}$ ohne Debatce in der Volkskammer nicht mehr enthalten waren.

21 Vgl. W. Bernet, Suasliche Lestung und Sicherung der Burgerrechte, fur. Disscrtation B, Jena 1978 (maschinenschriftich).

2) Vgl. Sozalisusche Demakraue (Zeitschrift) vom 22. Dez. 1972 (Beilage).

24 Geserz uber die ördichen Valksvertretungen und ihrer Organc in der Deurschen Demokrauschen Republik vom 12, Juli 1973 GBI. 1. S. jor. 
Die Verfassungsänderung vom 7. Okt. 1974 legre die Existenz von Beschwerdeausschüssen nicht mehr fest. Das Eingabengesecz vom 19. Juni $2975^{21}$ hob den Eingabenerlaß von 1969 auf und tilgte dic letztc legislative Spur dieser Gremien.

Wenn auch den Beschwerdeausschüssen kein dauerndes Schicksal beschieden war, so zeugr doch ihre Existenz davon, daß die Idee der Wahrung der Geserzlichkeir in der statetichen Verwaltung der DDR trotz großer Hindernisse und Verdikte nicht zum Erliegen gekommen ist. Letzelich hatte sich jedoch stets der mächtige Apparat der Verwaltungen einer förmlichen Kontrolle durch die Bürger, Gerichte oder gerichtsähnliche Instanzen entzogen. Die Unklarheiten zwischen Eingaben und Rechrsmitteln wurden bis in die letzten Monate nicht abgebaut. Auf die ungeheuercn Ausmaße insbesondere der Eingaben wurde verwiesen. Sie täuschten ein Demokratieverständ́nis vor, das von den Bürgern nicht wirkliches Rechesbewußtsein verlangte. Der Verwaltungsapparar blendere mit den Eingaben bürgerfreundliches Verhalten vor; letztlich legte er sich auch durch diese Handhabung lahm. Das bereits zitierte Gesetz über die Zuständigkeit und das Verfahren der Gerichte zur gerichtlichen Nachprüfung von Verwaltungsentscheidungen sollte - so die Intentionen von Verretern der Rechrswissenschaft - die unfruchtbare Praxis bei der Bearbeitung yon Eingaben und Rechismittein becnden und den Übergang zu einer Verwalrungsgerichtsbarkeit einleiten. ${ }^{26}$ Das hätte die Möglichkeit geboten, Verwvaltungen und Bürger der DDR an den Rechtsweg allmählich zu gewöbnen. Der Rechtsweg ist der besserc, weil gerechtere Weg. Er kann aber auch für dic Bürger härer sein, weil die auf ihm gesprochenen Urteile mit Eingaben nicht mehr anfechtbar sind. Nunmehr wird dieser Rechtsweg wohl nicht melır im Zuge von Reformen eingeführn, sondern durch Überstülpen einer fremden oder fremd gewordenen Rechrsordnung. Die Resultate solleen wir bald zu bilanzieren haben.

25 Geselz über die Eingaben der Bürger vom 19. Jun 1975 GBI. I, S. 461

26 Vgl. W. Brrnet, Rechusstaadichkeit - Wesentliche Existenziorm der DDR-Staatsvcrwaltung, in: Staat und Recht 2/1990, S. 11 t 